PROCEEDINGS OF THE

AMERICAN MATHEMATICAL SOCIETY

Volume 137, Number 7, July 2009, Pages 2233-2241

S 0002-9939(09)09843-8

Article electronically published on February 18, 2009

\title{
TROPICAL BASES BY REGULAR PROJECTIONS
}

\author{
KERSTIN HEPT AND THORSTEN THEOBALD
}

(Communicated by Bernd Ulrich)

\begin{abstract}
We consider the tropical variety $\mathcal{T}(I)$ of a prime ideal $I$ generated by the polynomials $f_{1}, \ldots, f_{r}$ and revisit the regular projection technique introduced by Bieri and Groves from a computational point of view. In particular, we show that $I$ has a short tropical basis of cardinality at most $r+\operatorname{codim} I+1$ at the price of increased degrees, and we provide a computational description of these bases.
\end{abstract}

\section{INTRODUCTION}

Given a field $K$ endowed with a non-trivial real valuation ord $: K \rightarrow \mathbb{R}_{\infty}:=$ $\mathbb{R} \cup\{\infty\}$, the valuation extends to any fixed algebraic closure $\bar{K}$. The tropical variety $\mathcal{T}(I)$ of an ideal $I \triangleleft K\left[x_{1}, \ldots, x_{n}\right]$ is defined as the topological closure of the set

$$
\operatorname{ord} \mathcal{V}(I)=\left\{\left(\operatorname{ord}\left(z_{1}\right), \ldots, \operatorname{ord}\left(z_{n}\right)\right): z \in \mathcal{V}(I)\right\} \subseteq \mathbb{R}^{n},
$$

where $\mathcal{V}(I)$ denotes the zero set of $I$ in $\left(\bar{K}^{*}\right)^{n}$. Tropical varieties have been the subject of intensive recent studies ([2, 4, 8, 9, 11]; see [10 for a general introduction).

A basis $\mathcal{F}=\left\{f_{1}, \ldots, f_{r}\right\}$ of $I$ is called a tropical basis of $I$ if $\bigcap_{i=1}^{r} \mathcal{T}\left(f_{i}\right)=$ $\mathcal{T}(I)$. Bogart, Jensen, Speyer, Sturmfels, and Thomas initiated the systematic computational investigation of tropical bases [2, 8, by providing Gröbner-related techniques for computing tropical bases as well as by providing lower bounds on the size. They consider the field of Puiseux series $K=\mathbb{C}\{\{t\}\}$ with the natural valuation and concentrate on the "constant coefficient case", i.e., $I \triangleleft \mathbb{C}\left[x_{1}, \ldots, x_{n}\right]$. As a lower bound, they show that for $1 \leq d \leq n$ there is a $d$-dimensional linear ideal $I$ in $\mathbb{C}\left[x_{1}, \ldots, x_{n}\right]$ such that any tropical basis of linear forms in $I$ has size at least $\frac{1}{n-d+1}\left(\begin{array}{l}n \\ d\end{array}\right)$.

In this paper we explain that by dropping the assumption on the degree of the polynomials there always exists a small tropical basis for a prime ideal $I$, thus contrasting that lower bound.

Theorem 1.1. Let $I \triangleleft K\left[x_{1}, \ldots, x_{n}\right]$ be a prime ideal generated by the polynomials $f_{1}, \ldots, f_{r}$. Then there exist $g_{0}, \ldots, g_{n-\operatorname{dim} I} \in I$ with

$$
\mathcal{T}(I)=\bigcap_{i=0}^{n-\operatorname{dim} I} \mathcal{T}\left(g_{i}\right)
$$

Received by the editors September 21, 2007, and, in revised form, September 29, 2008.

2000 Mathematics Subject Classification. Primary 13P10, 14 Q99.

Key words and phrases. Tropical geometry, tropical variety, tropical basis, Bieri-Groves Theorem. 
and thus $\mathcal{G}:=\left\{f_{1}, \ldots, f_{r}, g_{0}, \ldots, g_{n-\operatorname{dim} I}\right\}$ is a tropical basis for $I$ of cardinality $r+\operatorname{codim} I+1$.

In particular, this also implies the universal (i.e., independent of $\operatorname{dim} I$ ) bound of $n+1$ polynomials in the representation (2).

The statement comes as a consequence of the regular projection technique introduced by Bieri and Groves [1. The purpose of this paper is to revisit this approach from the computational point of view, with the goal to provide an explicit and constructive description of the resulting tropical bases. Specifically, we apply tropical elimination on a particular class of ideals; for a general treatment of tropical elimination see the recent papers of Sturmfels, Tevelev, and Yu [12, 13].

Based on this construction, we characterize the Newton polytopes of the polynomials $g_{i}$ in the tropical bases for the special case of ideals generated by two linear polynomials. The tradeoff between the cardinality and the degree of tropical bases in the general case is subject to further study.

We remark that Theorem 1.1 can be seen as a tropical analogue to the EisenbudEvans Theorem from classical algebraic geometry, which states that every algebraic set in $n$-space is the intersection of $n$ hypersurfaces [5].

This paper is structured as follows. In Section 2 we introduce the relevant notation from tropical geometry and their relation to valuations. In Section 3 we provide the computational treatment of regular projections and prove Theorem 1.1. Section 4 provides some results on the characterization of the resulting Newton polytopes of the basis polynomials.

\section{TROPICAL GEOMETRY}

For a field $K$, a real valuation is a map ord $: K \rightarrow \mathbb{R}_{\infty}=\mathbb{R} \cup\{\infty\}$ with $K \backslash\{0\} \rightarrow \mathbb{R}$ and $0 \mapsto \infty$ such that $\operatorname{ord}(a b)=\operatorname{ord}(a)+\operatorname{ord}(b)$ and $\operatorname{ord}(a+b) \geq$ $\min \{\operatorname{ord}(a), \operatorname{ord}(b)\}$. Thus ord $=-\log \|\cdot\|$ for a non-Archimedean norm $\|\cdot\|$ on $K$. Examples include $K=\mathbb{Q}$ with the $p$-adic valuation or the field $K=\mathbb{C}\{\{t\}\}$ of Puiseux series with the natural valuation. We can extend the valuation map to $\bar{K}$ (cf. 4]) and to $\bar{K}^{n}$ via

$$
\text { ord }: \bar{K}^{n} \rightarrow \mathbb{R}_{\infty}^{n}, \quad\left(a_{1}, \ldots, a_{n}\right) \mapsto\left(\operatorname{ord}\left(a_{1}\right), \ldots, \operatorname{ord}\left(a_{n}\right)\right)
$$

We always assume that ord is non-trivial, i.e., $\operatorname{ord}\left(\bar{K}^{*}\right) \neq\{0\}$. Then the image $\operatorname{ord}\left(\bar{K}^{*}\right)$ is dense in $\mathbb{R}$.

Tropical polynomials are polynomials over the tropical semiring $\left(\mathbb{R}_{\infty}, \min ,+\right)$. For any polynomial $f=\sum_{\alpha} c_{\alpha} x^{\alpha} \in K\left[x_{1}, \ldots, x_{n}\right]$, the tropicalization of $f$ is the tropical polynomial defined by

$$
\operatorname{trop}(f)=\min _{\alpha}\left\{\operatorname{ord}\left(c_{\alpha}\right)+\alpha_{1} x_{1}+\cdots+\alpha_{n} x_{n}\right\},
$$

and the tropical hypersurface of $f$ is

$\mathcal{T}(f)=\left\{w \in \mathbb{R}^{n}\right.$ : the minimum in $\operatorname{trop}(f)$ is attained at least twice in $\left.w\right\}$. For an ideal $I \triangleleft K\left[x_{1}, \ldots, x_{n}\right]$, the tropical variety of $I$ can be defined either by

$$
\mathcal{T}(I)=\bigcap_{f \in I} \mathcal{T}(f)
$$

or equivalently by (1); see 44. Note that we consider tropical varieties from a set-theoretic point of view; see [12] for a more refined treatment of the underlying multiplicities. 
We shortly review the link between tropical geometry and classical valuation theory. For a prime ideal $I$, let $A:=K\left[x_{1}, \ldots, x_{n}\right] / I$ be its coordinate ring. It is well known (see, e.g., [6]) that each valuation on $K$ can be extended to a valuation on $A$. Let $\Delta_{A}^{\text {ord }}$ be defined by

$$
\Delta_{A}^{\text {ord }}=\left\{\left(w\left(x_{1}\right), \ldots, w\left(x_{n}\right)\right) \in \mathbb{R}^{n} \mid w: A \rightarrow \mathbb{R}_{\infty} \text { a valuation with }\left.w\right|_{K}=\text { ord }\right\} .
$$

This subset of $\mathbb{R}^{n}$ coincides with the tropical variety of $I$,

$$
\Delta_{A}^{\text {ord }}=\mathcal{T}(I)
$$

(see [4). Bieri and Groves [1] showed that $\Delta_{A}^{\text {ord }}$ (and thus $\mathcal{T}(I)$ as well) is a pure polyhedral complex of dimension equal to the transcendence degree of $A$ over $K$ and rationally defined over the value group ord $\left(K^{*}\right)$ of ord.

\section{Projections and the main theorem}

Let $I \triangleleft K\left[x_{1}, \ldots, x_{n}\right]$ be an $m$-dimensional prime ideal. The main geometric idea is to consider $n-m+1$ different (rational) projections $\pi_{0}, \ldots, \pi_{n-m}: \mathbb{R}^{n} \rightarrow \mathbb{R}^{m+1}$. If these projections are sufficiently generic (as specified below), then we obtain

$$
\bigcap_{i=0}^{n-m} \pi_{i}^{-1}\left(\pi_{i}(\mathcal{T}(I))\right)=\mathcal{T}(I)
$$

and each of the sets $\pi_{i}^{-1}\left(\pi_{i}(\mathcal{T}(I))\right)$ is a tropical hypersurface.

First we consider the image of the tropical variety $\mathcal{T}(I)$ under a single (rational) projection

$$
\begin{aligned}
\pi: \mathbb{R}^{n} & \rightarrow \mathbb{R}^{m+1}, \\
x & \mapsto A x
\end{aligned}
$$

with a non-singular rational matrix $A$ whose rows are denoted by $a^{(1)}, \ldots, a^{(m+1)}$. Let $u^{(1)}, \ldots, u^{(l)} \in \mathbb{Z}^{n}$ with $l:=n-(m+1)$ be a basis of the orthogonal complement of $\operatorname{span}\left\{a^{(1)}, \ldots, a^{(m+1)}\right\}$.

Set $R=K\left[x_{1}, \ldots, x_{n}, \lambda_{1}, \ldots, \lambda_{l}\right]$, and for any polynomial $f \in K\left[x_{1}, \ldots, x_{n}\right]$ let $\hat{f}$ be the composition of $f$ with the monomial map $x_{i} \mapsto x_{i} \prod_{j=1}^{l} \lambda_{j}{ }^{u_{i}^{(j)}}$, i.e.,

$$
\hat{f}\left(x_{1}, \ldots, x_{n}, \lambda_{1}, \ldots, \lambda_{l}\right)=f\left(x_{1} \prod_{j=1}^{l} \lambda_{j}^{u_{1}^{(j)}}, \ldots, x_{n} \prod_{j=1}^{l} \lambda_{j}{ }^{u_{n}^{(j)}}\right) \in R .
$$

Define the ideal $J \triangleleft R$ by

$$
J=\langle\hat{f} \in R: f \in I\rangle .
$$

We show the following characterization of $\pi^{-1}(\pi(\mathcal{T}(I)))$ in terms of elimination.

Theorem 3.1. Let $I \triangleleft K\left[x_{1}, \ldots, x_{n}\right]$ be an $m$-dimensional prime ideal and $\pi$ : $\mathbb{R}^{n} \rightarrow \mathbb{R}^{m+1}$ be a rational projection. Then $\pi^{-1}(\pi(\mathcal{T}(I)))$ is a tropical variety with

$$
\pi^{-1}(\pi(\mathcal{T}(I)))=\mathcal{T}\left(J \cap K\left[x_{1}, \ldots, x_{n}\right]\right) .
$$

In order to prove Theorem 3.1 we first consider algebraically regular projections (as defined below). At the end of this section we also cover the remaining special cases.

We start with an auxiliary statement which holds for an arbitrary rational projection $\pi$. 
Lemma 3.2. For any $w \in \mathcal{T}\left(J \cap K\left[x_{1}, \ldots, x_{n}\right]\right)$ and $u \in \operatorname{span}\left\{u^{(1)}, \ldots, u^{(l)}\right\}$ we have $w+u \in \mathcal{T}\left(J \cap K\left[x_{1}, \ldots, x_{n}\right]\right)$.

Proof. Let $u=\sum_{i=1}^{l} \mu_{j} u^{(j)}$ with $\mu_{1}, \ldots, \mu_{l} \in \mathbb{Q}$. The case of real $\mu_{i}$ then follows as well.

Let $w \in \mathcal{T}\left(J \cap K\left[x_{1}, \ldots, x_{n}\right]\right)$. Since $\mathcal{T}\left(J \cap K\left[x_{1}, \ldots, x_{n}\right]\right)$ is closed, we can assume without loss of generality that there exists $z \in \mathcal{V}\left(J \cap K\left[x_{1}, \ldots, x_{n}\right]\right)$ with ord $z=w$. Define $y=\left(y^{\prime}, y^{\prime \prime}\right) \in\left(\bar{K}^{*}\right)^{n+l}$ by

$$
y=\left(y^{\prime}, y^{\prime \prime}\right)=\left(z_{1} t^{\sum_{j=1}^{l} \mu_{j} u_{1}^{(j)}}, \ldots, z_{n} t^{\sum_{j=1}^{l} \mu_{j} u_{n}^{(j)}}, t^{-\mu_{1}}, \ldots, t^{-\mu_{l}}\right) .
$$

For any $f \in I$, the point $y$ is a zero of the polynomial $\hat{f}$ in the ring $R$, and thus $y \in \mathcal{V}(J)$. Hence, $y^{\prime} \in \mathcal{V}\left(J \cap K\left[x_{1}, \ldots, x_{n}\right]\right)$. Moreover,

$$
\operatorname{ord} y^{\prime}=\left(w_{1}+\sum_{j=1}^{l} \mu_{j} u_{1}^{(j)}, \ldots, w_{n}+\sum_{j=1}^{l} \mu_{j} u_{n}^{(j)}\right)=w+\sum_{j=1}^{l} \mu_{j} u^{(j)}=w+u,
$$

which proves our claim.

Lemma 3.3. Let $I \triangleleft K\left[x_{1}, \ldots, x_{n}\right]$ be an ideal. Then $J \cap K\left[x_{1}, \ldots, x_{n}\right] \subseteq I$.

Proof. Let $p=\sum_{i} h_{i} \hat{f}_{i}$ be a polynomial in $J \cap K\left[x_{1}, \ldots, x_{n}\right]$ with $f_{i} \in I$. Since $p$ is independent of $\lambda_{1}, \ldots, \lambda_{l}$ we have

$$
p=\left.p\right|_{\lambda_{1}=1, \ldots, \lambda_{l}=1}=\left.\sum_{i} h_{i}\right|_{\lambda_{1}=1, \ldots, \lambda_{l}=1} f_{i} \in I .
$$

We call a rational projection algebraically regular for $I$ if for each $i \in\{1, \ldots, l\}$ the elimination ideal $J \cap K\left[x_{1}, \ldots, x_{n}, \lambda_{1}, \ldots, \lambda_{i}\right]$ has a finite basis $\mathcal{F}_{i}$ such that in every polynomial $f \in \mathcal{F}_{i}$ the coefficients of the powers of $\lambda_{i}$ (when considering $f$ as a polynomial in $\lambda_{i}$ ) are monomials in $x_{1}, \ldots, x_{n}, \lambda_{1}, \ldots, \lambda_{i-1}$.

The following statement shows that the set of algebraically regular projections is dense in the set of all real projections $\pi: \mathbb{R}^{n} \rightarrow \mathbb{R}^{m+1}$.

Lemma 3.4. The set of projections which are not algebraically regular is contained in a finite union of hyperplanes within the space of all projections $\pi: \mathbb{R}^{n} \rightarrow \mathbb{R}^{m+1}$.

Proof. It suffices to show that for the choice of $u^{(l)}$, we just have to avoid a lowerdimensional subset of $\mathbb{R}^{n} \backslash\{0\}$. For $u^{(1)}, \ldots, u^{(l-1)}$ we can then argue inductively (however, an explicit description then becomes more involved). Assume that $I$ is generated by $f_{1}, \ldots, f_{s}$. Then $J=\left\langle\hat{f}_{j}: 1 \leq j \leq s\right\rangle$. For any fixed $j$, the polynomial $\hat{f}_{j}$ is of the form

$$
\hat{f}_{j}=\sum_{\alpha \in \mathcal{A}_{j}} c_{\alpha} x^{\alpha} \lambda_{1}^{\sum \alpha_{i} u_{i}^{(1)}} \cdots \lambda_{l}^{\sum \alpha_{i} u_{i}^{(l)}}
$$

with $\mathcal{A}_{j} \subset \mathbb{Z}^{n}$ finite. Thus all $\lambda_{l}^{k}$ have monomial coefficients if

$$
\sum \alpha_{i} u_{i}^{(l)} \neq \sum \beta_{i} u_{i}^{(l)}
$$

for all $\alpha, \beta \in \mathcal{A}_{j}$ with $\alpha \neq \beta$. So we have to choose $u^{(l)}$ from the subset

$$
\bigcap_{j}\left\{u \in \mathbb{R}^{n}: \sum \alpha_{i} u_{i}^{(l)} \neq \sum \beta_{i} u_{i}^{(l)} \text { for all } \alpha, \beta \in \mathcal{A}_{j} \text { with } \alpha \neq \beta\right\} \text {. }
$$


Hence, the algebraically non-regular projections are contained in a finite number of hyperplanes.

Theorem 3.5. Let $I \triangleleft K\left[x_{1}, \ldots, x_{n}\right]$ be a prime ideal and $\pi: \mathbb{R}^{n} \rightarrow \mathbb{R}^{m+1}$ be an algebraically regular projection. Then $\pi^{-1} \pi(\mathcal{T}(I))$ is a tropical variety with

$$
\pi^{-1} \pi(\mathcal{T}(I))=\mathcal{T}\left(J \cap K\left[x_{1}, \ldots, x_{n}\right]\right) .
$$

Proof. Let $w \in \pi^{-1} \pi(\mathcal{T}(I))$. Since the right-hand set of (4) is closed, we can assume without loss of generality that there exists $z^{\prime} \in \mathcal{V}(I)$ and $u \in \operatorname{span}\left\{u^{(1)}, \ldots, u^{(l)}\right\}$ with ord $z^{\prime}=w+u$. For any $f \in I$, the point

$$
z:=\left(z^{\prime}, 1\right)
$$

is a zero of the polynomial $\hat{f} \in R$, and thus $z \in \mathcal{V}(J)$. Hence, $z^{\prime} \in \mathcal{V}(J \cap$ $\left.K\left[x_{1}, \ldots, x_{n}\right]\right)$. By Lemma 3.2, $w \in \mathcal{T}\left(J \cap K\left[x_{1}, \ldots, x_{n}\right]\right)$ as well.

Now let $w \in \mathcal{T}\left(J \cap K\left[x_{1}, \ldots, x_{n}\right]\right)$. Again we can assume that there is a $z \in \mathcal{V}\left(J \cap K\left[x_{1}, \ldots, x_{n}\right]\right) \subseteq\left(\bar{K}^{*}\right)^{n}$ with $w=\operatorname{ord}(z)$. The projection is algebraically regular, which means that the generators of the elimination ideals $J \cap$ $K\left[x_{1}, \ldots, x_{n}, \lambda_{1}, \ldots, \lambda_{i}\right]$ have only monomials as coefficients with respect to $\lambda_{i}$. By the Extension Theorem (see, e.g., [3]), we can extend the root $z$ inductively to a root $\tilde{z} \in \mathcal{V}(J)$ with the same first $n$ entries. The definition of $J$ says that

$$
z^{\prime}:=\left(z_{1} \tilde{z}_{n+1}^{u_{1}^{(1)}} \cdots \tilde{z}_{n+l}^{u_{1}^{(l)}}, \ldots, z_{n} \tilde{z}_{n+1}^{u_{n}^{(1)}} \cdots \tilde{z}_{n+l}^{u_{n}^{(l)}}\right)
$$

is a root of $I$. Then

$$
\operatorname{ord}\left(z^{\prime}\right)=\operatorname{ord}(z)+\sum_{i=1}^{l} \operatorname{ord}\left(\tilde{z}_{n+i}\right) u^{(i)},
$$

which means that $\operatorname{ord}(z)=w \in \pi^{-1} \pi(\mathcal{T}(I))$.

This completes the proof of Theorem 3.1 for the case of algebraically regular projections.

In the following, we consider the notion of geometric regularity.

Definition 1. Let $\mathcal{C}$ be a polyhedral complex in $\mathbb{R}^{n}$. A projection $\pi: \mathbb{R}^{n} \rightarrow \mathbb{R}^{m+1}$ is called geometrically regular if the following two conditions hold.

(1) For any $k$-face $\sigma$ of $\mathcal{C}$ we have $\operatorname{dim}(\pi(\sigma))=k, 0 \leq k \leq \operatorname{dim} \mathcal{C}$.

(2) If $\pi(\sigma) \subseteq \pi(\tau)$, then $\sigma \subseteq \tau$ for all $\sigma, \tau \in \mathcal{C}$.

These conditions ensure that we can recover the whole complex $\mathcal{C}$ from the projections.

Corollary 3.6. In the situation of Theorem 3.5, if $\operatorname{dim} \pi(\mathcal{T}(I))=m$, then $\pi^{-1} \pi(\mathcal{T}(I))$ is a tropical hypersurface.

In particular, this holds when the projection is geometrically regular.

Proof. $\operatorname{dim} \pi^{-1} \pi(\mathcal{T}(I))=\operatorname{dim} \pi(\mathcal{T}(I))+\operatorname{dim} \operatorname{ker} \pi=m+(n-(m+1))=n-1$.

Let $I \triangleleft K\left[x_{1}, \ldots, x_{n}\right]$ be a prime ideal and $m=\operatorname{dim} I$. Then $\mathcal{T}(I)$ is a pure $m$ dimensional polyhedral complex. Bieri and Groves [1] used the following geometric technique (which actually was also used to prove that $\mathcal{T}(I)$ has this polyhedral property).

There exists a finite family $\mathcal{X}=\left\{\mathcal{X}_{1}, \ldots, \mathcal{X}_{s}\right\}$ of $m$-dimensional affine subspaces with $\mathcal{T}(I) \subseteq \bigcup_{i=1}^{s} \mathcal{X}_{s}$. By the finiteness of $\mathcal{X}$, for a sufficiently generic choice of 
$n-m+1$ geometrically regular projections $\pi_{0}, \ldots, \pi_{n-m}$, the set-theoretic intersection of the inverse projections exactly yields the original polyhedral complex. This follows from [1, Thm. 4.4] (and its proof) in connection with the pure-dimensionality of $\mathcal{T}(I)$.

Proposition 3.7 (Bieri, Groves [1]). Let $I \triangleleft K\left[x_{1}, \ldots, x_{n}\right]$ be a prime ideal. For any dense set $\mathcal{D}$ of projections there exist $\operatorname{codim} I+1$ projections $\pi_{0}, \ldots, \pi_{\operatorname{codim} I} \in \mathcal{D}$ such that

$$
\mathcal{T}(I)=\bigcap_{i=0}^{\operatorname{codim} I} \pi_{i}^{-1} \pi_{i}(\mathcal{T}(I)) .
$$

By Lemma 3.4 the set of algebraically regular projections is dense in the space of projections. Hence, combining Proposition 3.7 with Theorem 3.5 yields Theorem 1.1. Note that by Lemma 3.3, the generators $g_{i}$ are actually contained in $I$.

Using this knowledge about the existence of some tropical basis, we can also provide the proof of Theorem 3.1 for arbitrary rational projections.

Theorem 3.8 (Tropical Extension Theorem). Let $I \triangleleft K\left[x_{0}, \ldots, x_{n}\right]$ be an ideal and $I_{1}=I \cap K\left[x_{1}, \ldots, x_{n}\right]$ be its first elimination ideal. For any $w \in \mathcal{T}\left(I_{1}\right)$ there exists a point $\tilde{w}=\left(w_{0}, \ldots, w_{n}\right) \in \mathbb{R}^{n+1}$ with $w_{i}=\tilde{w}_{i}$ for $1 \leq i \leq n$ and $\tilde{w} \in \mathcal{T}(I)$.

Proof. First let $w \in \operatorname{ord}\left(\mathcal{V}\left(I_{1}\right)\right)$, so that there exists $z \in \mathcal{V}\left(I_{1}\right)$ with $\operatorname{ord}(z)=w$. Let $\mathcal{G}=\left\{g_{1}, \ldots, g_{s}\right\}$ be a reduced Gröbner basis of $I$ with respect to a lexicographical term order with $x_{0}>x_{i}, 1 \leq i \leq n$, i.e.,

$$
g_{i}=h_{i}\left(x_{1}, \ldots, x_{n}\right) x_{0}^{\operatorname{deg}_{x_{0}} g_{i}}+\text { terms of lower degree in } x_{0} .
$$

There are two cases to consider:

Case 1. $z \notin \mathcal{V}\left(h_{1}, \ldots, h_{s}\right)$. Then by the classical Extension Theorem there is a root $\tilde{z}$ of $I$ which extends $z$, so ord $(\tilde{z})=: \tilde{w}$ extends $w$.

Case 2. $z \in \mathcal{V}\left(h_{1}, \ldots, h_{s}\right)$. Then $w=\operatorname{ord}(z) \in \mathcal{T}\left(h_{1}, \ldots, h_{s}\right)$. Let $\mathcal{P}=\left\{p_{1}, \ldots, p_{t}\right\}$ be a tropical basis of $I$.

Let $p_{j}$ be any of these polynomials. $p_{j}$ has the form

$$
p_{j}=q_{j}\left(x_{1}, \ldots, x_{n}\right) x_{0}^{\operatorname{deg}_{x_{0}} p_{j}}+\text { terms of lower degree in } x_{0} .
$$

Since $\mathcal{G}$ is a lexicographic Gröbner basis, we have $q_{j}\left(x_{1}, \ldots, x_{n}\right)=: \sum k_{\alpha} x^{\alpha} \in$ $\left\langle h_{1}, \ldots, h_{s}\right\rangle$. Hence, the minimum

$$
\min _{\alpha}\left\{\operatorname{ord}\left(k_{\alpha}\right)+\alpha_{1} x_{1}+\cdots+\alpha_{n} x_{n}\right\}
$$

is attained twice at $w$. We can pick a sufficiently small value $w_{0}^{(j)} \in \mathbb{R}$ so that all terms $x_{1}^{m_{1}} \cdots x_{n}^{m_{n}} x_{0}^{m_{0}}$ of $p_{j}$ with $m_{0}<\operatorname{deg}_{x_{0}} p_{j}$ have a larger value $m_{1} w_{1}+\cdots+$ $m_{n} w_{n}+m_{0} w_{0}^{(j)}$. But then the minimum of all values of all terms of $p_{j}$ is attained at least twice; it is

$$
\min _{\alpha}\left\{\operatorname{ord}\left(k_{\alpha}\right)+\alpha_{1} w_{1}+\cdots+\alpha_{n} w_{n}\right\}+\operatorname{deg}_{x_{0}} p_{j} \cdot w_{0}^{(j)} .
$$

So $\left(w_{0}^{(j)}, w_{1}, \ldots, w_{n}\right) \in \mathcal{T}\left(h_{j}\right)$.

By setting $w_{0}=\min _{j}\left\{w_{0}^{(j)}\right\}$ and $\tilde{w}:=\left(w_{0}, \ldots, w_{n}\right) \in \mathcal{T}(I)$, we obtain the desired extension of $w$. This completes case 2 . 
Now let $w=\lim _{i \rightarrow \infty} w^{(i)}$ be in the closure of $\operatorname{ord}\left(\mathcal{V}\left(I_{1}\right)\right)$. Then there exist $\tilde{w}^{(i)} \in \mathcal{T}(I)$ with $\tilde{w}_{j}^{(i)}=w_{j}^{(i)}$ for $1 \leq j \leq n$. Let $\mathcal{P}=\left\{p_{1}, \ldots, p_{t}\right\}$ be again a tropical basis of $I$. Then we can assume w.l.o.g. that the minimum in $\operatorname{trop}\left(p_{k}\right), 1 \leq k \leq t$, for $\tilde{w}^{(i)}$ is attained at the same terms. This gives us conditions for the $\tilde{w}_{0}^{(i)}$ :

$$
\left.k^{(i)} \leq \tilde{w}_{0}^{(i)} \leq l^{(i)} \quad \text { (one of them can be } \pm \infty\right) .
$$

These bounds vary continuously with $w^{(i)}$. So we can choose $\tilde{w}_{0}$ arbitrarily in $\left[\lim k^{(i)}, \lim l^{(i)}\right]$ (only one of the limits can be $\pm \infty$ ).

\section{The Newton polytopes for the linear case}

As mentioned earlier, an ideal generated by linear forms may not have a small tropical basis if we restrict the basis to consist of linear forms. Using our results from Section 3 we can provide a short basis at the price of increased degrees. A natural question is to provide a good characterization for the Newton polytopes of the resulting basis polynomials. Here, we briefly discuss the special case of a prime ideal $I$ generated by two linear polynomials $f=\sum_{i=1}^{n} a_{i} x_{i}+a_{n+1}, g=$ $\sum_{i=1}^{n} b_{i} x_{i}+b_{n+1} \in K\left[x_{1}, \ldots, x_{n}\right]$ and kernel direction $v$. We assume $v \in \mathbb{Z}_{+}^{3}$.

In order to characterize the Newton polytope of the additional polynomials in the tropical basis, we consider the resultant of the polynomials $\hat{f}, \hat{g}$ :

$$
\begin{aligned}
& \hat{f}=a_{1} x_{1} \lambda^{v_{1}}+\cdots+a_{n} x_{n} \lambda^{v_{n}}+a_{n+1}, \\
& \hat{g}=b_{1} x_{1} \lambda^{v_{1}}+\cdots+b_{n} x_{n} \lambda^{v_{n}}+b_{n+1}
\end{aligned}
$$

in $K\left[x_{1}, \ldots, x_{n}, \lambda\right]$. Suppose that the components $v_{i}$ are distinct. Then w.l.o.g. we can assume $v_{1}>v_{2}>\cdots>v_{n}>v_{n+1}:=0$.

In order to apply the results of Gel'fand, Kapranov and Zelevinsky [7] on the Newton polytope of the resultant, we consider the representation

$$
\operatorname{Res}_{\lambda}(\hat{f}, \hat{g})=\sum_{p, q} c_{p, q} a^{p} b^{q} x_{1}^{p_{1}+q_{1}} \cdots x_{n}^{p_{n}+q_{n}}
$$

with $p=\left(p_{1}, \ldots, p_{n+1}\right), q=\left(q_{1}, \ldots, q_{n+1}\right) \in \mathbb{Z}_{+}^{n+1}$. Regarding (5) as a polynomial in $a_{1}, \ldots, a_{n+1}, b_{1}, \ldots, b_{n+1}$, the Newton polytope is contained in the set $\mathcal{Q}_{n} \subset$ $\mathbb{Z}^{2 n+2}$ of non-negative integer points $(p, q)$ with

(1) $\sum_{i=1}^{n+1} p_{i}=\sum_{j=1}^{n+1} q_{j}=v_{1}$

(2) $\sum_{i=1}^{n+1} v_{i} p_{i}+\sum_{j=1}^{n+1} v_{j} q_{j}=v_{1}^{2}$,

(3) $\sum_{\substack{1 \leq k \leq n \\ 0 \leq v_{1}-v_{k} \leq i}}\left(i-v_{1}+v_{k}\right) p_{k}+\sum_{\substack{1 \leq l \leq n \\ 0 \leq v_{1}-v_{l} \leq j}}\left(j-v_{1}+v_{l}\right) q_{l} \geq i j \quad\left(0 \leq i, j \leq v_{1}\right)$.

Hence, we can conclude:

Corollary 4.1. The set of integer points in the Newton polytope $\operatorname{New}\left(\operatorname{Res}_{\lambda}(\hat{f}, \hat{g})\right)$ $\subset \mathbb{Z}^{n}$ is contained in the image of $\mathcal{Q}_{n}$ under the mapping

$$
\left(p_{1}, \ldots, p_{n+1}, q_{1}, \ldots, q_{n+1}\right) \mapsto\left(p_{1}+q_{1}, \ldots, p_{n}+q_{n}\right) .
$$

Example 4.2. Let $f=2 x+y-4, g=x+2 y+z-1$ and $I \triangleleft K[x, y, z]$ be the ideal generated by $f$ and $g$. Further let $\operatorname{ord}(\cdot)$ be the 2 -adic valuation (see Figure 1 for a figure of $\mathcal{T}(I))$. 


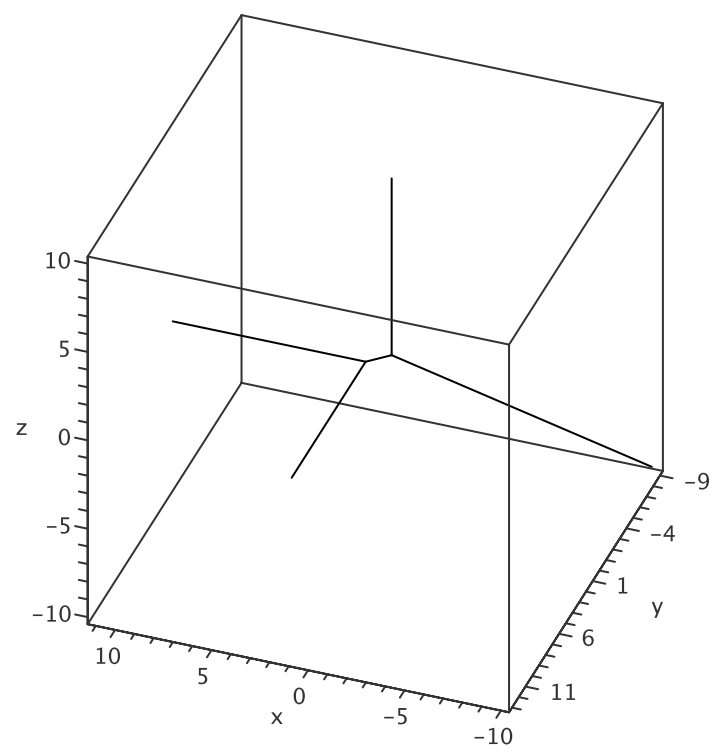

Figure 1. Tropical line $\mathcal{T}(I)$ in 3 -space

Actually, the first projection can be chosen arbitrarily (even geometrically nonregular). We choose a projection $\pi_{1}$ whose kernel is generated by $(0,0,1)$. Then the tropical hypersurface $\pi_{1}^{-1} \pi_{1}(\mathcal{T}(I))$ is obviously generated by the first polynomial, i.e. $\pi_{1}^{-1} \pi_{1}(\mathcal{T}(I))=\mathcal{T}(2 x+y-4)$, and the Newton polytope of that polynomial is a triangle (so the projection is geometrically non-regular).

We choose $\pi_{2}$ as the projection with kernel generated by $v=(1,2,0)$. Since $\operatorname{Res}_{\lambda}(\hat{f}, \hat{g})=y \cdot\left(6 x^{2}+6 x^{2} z+49 y+14 y z+y z^{2}\right)$, we obtain $\pi_{2}^{-1} \pi_{2}(\mathcal{T}(I))=\mathcal{T}\left(6 x^{2}+\right.$ $\left.6 x^{2} z+49 y+14 y z+y z^{2}\right)$. The intersection of $\pi_{1}^{-1} \pi_{1}(\mathcal{T}(I))$ and $\pi_{2}^{-1} \pi_{2}(\mathcal{T}(I))$ is one-dimensional.

For the last projection $\pi_{3}$ we choose a projection whose kernel is generated by $(3,2,1)$. In this case, the resultant is $x\left(8 x^{2}+147 y^{3}+16 x z^{3}-76 x y z-12 y^{2} z^{2}\right)$, and hence $\pi_{3}^{-1} \pi_{3}(\mathcal{T}(I))=\mathcal{T}\left(8 x^{2}+147 y^{3}+16 x z^{3}-76 x y z-12 y^{2} z^{2}\right)$. For $\pi_{3}$, all possible vectors $\left(p_{1}+q_{1}, p_{2}+q_{2}, p_{3}+q_{3}\right)$ of Corollary 4.1 are

$$
\{(3,0,0),(2,1,1),(2,0,3),(1,3,0),(1,2,2)\},
$$

and this set coincides with the support of the resultant.

The Newton polytopes of the last two of the three tropical hypersurfaces are quadrangles. Adding the three non-linear polynomials to the basis of $I$ yields a tropical basis.

\section{ACKNOWLEDGMENTS}

We thank Robert Bieri, Tristram Bogart, Jan Draisma, Bernd Sturmfels, and an anonymous referee for useful comments. 


\section{REFERENCES}

1. R. Bieri and J. R. J. Groves, The geometry of the set of characters induced by valuations, J. Reine Angew. Math. 347 (1984), 168-195. MR733052 (86c:14001)

2. T. Bogart, A. N. Jensen, D. Speyer, B. Sturmfels, and R. R. Thomas, Computing tropical varieties, J. Symb. Comp. 42 (2007), no. 1-2, 54-73. MR2284285 (2007j:14103)

3. D. Cox, J. Little, and D. O'Shea, Ideals, varieties, and algorithms: An introduction to computational algebraic geometry and commutative algebra, 3rd ed., Springer-Verlag, New York, 2007. MR2290010 (2007h:13036)

4. M. Einsiedler, M. M. Kapranov, and D. Lind, Non-Archimedean amoebas and tropical varieties, J. Reine Angew. Math. 601 (2006), 139-157. MR2289207(2007k:14038)

5. D. Eisenbud and E. G. Evans, Jr., Every algebraic set in $n$-space is the intersection of $n$ hypersurfaces, Invent. Math. 19 (1973), 107-112. MR0327783 (48:6125)

6. O. Endler, Valuation theory, Universitext, Springer-Verlag, New York, 1972. MR0357379 (50:9847)

7. I. M. Gel'fand, M. M. Kapranov, and A. V. Zelevinsky, Newton polytopes of the classical resultant and discriminant, Adv. Math. 84 (1990), 237-254. MR.1080979 (92a:14060)

8. A. N. Jensen, Algorithmic aspects of Gröbner fans and tropical varieties, Ph.D. thesis, University of Aarhus, 2007.

9. A. N. Jensen, H. Markwig, and T. Markwig, An algorithm for lifting points in a tropical variety, Collect. Math. 59 (2008), no. 2, 129-165. MR2414142(2009a:14077)

10. J. Richter-Gebert, B. Sturmfels, and T. Theobald, First steps in tropical geometry, Idempotent Mathematics and Mathematical Physics (V.P. Maslov and G.L. Litvinov, eds.), Contemp. Math., vol. 377, Amer. Math. Soc., Providence, RI, 2005, 289-317. MR2149011(2006d:14073)

11. D. Speyer and B. Sturmfels, The tropical Grassmannian, Adv. Geom. 4 (2004), no. 3, 389-411. MR:2071813 (2005d:14089)

12. B. Sturmfels and J. Tevelev, Elimination theory for tropical varieties, Math. Res. Lett. 15 (2008), no. 3, 543-562. MR2407231

13. B. Sturmfels and J. Yu, Tropical implicitization and mixed fiber polytopes, Software for Algebraic Geometry (N. Takayama, M. Stillman and J. Verschelde, eds.), IMA Volumes in Mathematics and Its Applications, vol. 148, Springer, New York, 2008, pp. 111-132.

FB 12 - Institut für Mathematik, J.W. Goethe-Universität, Postfach 111932, D-60054 Frankfurt am Main, Germany

E-mail address: hept@math.uni-frankfurt.de

FB 12 - Institut für Mathematik, J.W. Goethe-Universität, Postfach 111932, D-60054 Frankfurt am Main, Germany

E-mail address: theobald@math.uni-frankfurt.de 